\title{
THE HEAT EQUATION IN SEVERAL COMPLEX VARIABLES
}

\author{
BY NANCY K. STANTON ${ }^{1}$
}

Introduction. I will discuss some recent progress and open questions for two problems about the heat equation in several complex variables. These problems are analogues of some classical problems in differential geometry. Both in the problems in several complex variables and the classical problems, one has a differential operator $\boldsymbol{P}$ on a manifold $M$-classically the Laplace-Beltrami operator. One wishes to use the fundamental solution $p(x, y, t)$ of the initial value problem for the corresponding heat equation

$$
(\partial / \partial t+P) u=0
$$

to study relationships between the spectrum of $P$ and the geometry and topology of $M$. From an explicit construction of $p$, one tries to obtain an asymptotic expansion of the trace of $P$ as $t \rightarrow 0$. Then a geometric interpretation of the coefficients allows one to relate the spectrum and the geometry. Because the problems in several complex variables are not elliptic, classical methods for constructing the fundamental solution $p$ do not apply. Thus, the problem of constructing $p$ in these situations is new and interesting.

In $\S \S 1$ and 2 I will survey some results about the heat equation in Riemannian and Hermitian geometry, with applications to spectral geometry. This survey is not intended to be complete, but to give some idea of the kinds of results which have been obtained by heat equation methods, to suggest some of the remaining open problems, and to motivate the work in several complex variables. The surveys by Berger $[\mathbf{B}]$ and Singer [Si] contain a more complete description of the earlier results in the subject. After this survey, I will discuss the two analogous problems in several complex variables. In \$3 I will discuss the heat equation for the $\bar{\partial}_{b}$-Laplacian on the boundary of a strictly pseudoconvex domain in $\mathbf{C}^{n}$, and in $\$ 4 \mathrm{I}$ will discuss the heat equation for the $\bar{\partial}$-Neumann problem in a strictly pseudoconvex domain in $\mathbf{C}^{n}$. I will discuss only the simplest case of each problem, and will give a fairly complete survey of what is known for this case. More general cases are handled in the references for $\$ \S 3$ and 4.

This is an expanded version of an invited address that the author gave at the American Mathematical Society Meeting at Bryn Mawr on March 16, 1982; received by the editors December 1, 1983.

1980 Mathematics Subject Classification. Primary 32F20, 58G11.

\footnotetext{
${ }^{1}$ Research supported in part by NSF Grant MCS82-00442, the Alfred P. Sloan Foundation and the Max-Planck-Institut für Mathematik.
} 
1. The heat equation in Riemannian geometry. Let $M$ be a compact oriented Riemannian manifold of dimension $n$. The heat equation for $p$ forms on $M$ is

$$
\partial u / \partial t+\Delta u=0
$$

where $u$ is a $p$ form depending on $t \in \mathbf{R}^{+}$and $\Delta=d d^{*}+d^{*} d$ is the LaplaceBeltrami operator. Note that $\Delta$ is a positive operator, whereas the usual Euclidean Laplacian $\sum \partial^{2} / \partial x_{i}^{2}$ is a negative operator, so I need the plus sign in (1.1). A $p$ form $u$ depending on $t \in \mathbf{R}^{+}$solves the initial value problem for the heat equation with inital value $u_{0} \in C(M)$ if $u$ satisfies (1.1) and

$$
\lim _{t \rightarrow 0} u(x, t)=u_{0}(x) \text {. }
$$

The initial value problem for the heat equation on functions on compact oriented Riemannian manifolds was first solved by Minakshisundaram and Pleijel [MP]. On forms it was solved by Milgram and Rosenbloom [MR], and later more explicitly by Gaffney [Ga] using the methods of [MP]. The solution of the initial value problem is obtained by applying the heat operator $e^{-t \Delta}$, the semigroup generated by $-\Delta$, to the initial data. The heat operator is given by integration over $M$ against a smooth kernel $p(x, y, t)$ on $M \times M \times \mathbf{R}^{+}$. On functions, to first approximation the kernel looks like the "Gaussian" kernel

$$
(1 / 4 \pi t)^{n / 2} e^{-r^{2} / 4 t},
$$

where $r$ is the Riemannian distance from $x$ to $y$. Minakshisundaram and Pleijel construct the kernel quite explicitly. The construction involves the iterative solution of an appropriate integral equation. The convergence of the resulting series follows in a straightforward way from properties of the Gaussian kernel.

The primary motivation in studying the heat equation has been to obtain results on the spectral geometry of the manifold. One type of application is given by Milgram and Rosenbloom, who used the heat equation to give an alternate proof of the existence of a harmonic form in a given de Rham cohomology class: if $u_{0}$ is a closed form and $u$ is the solution of the heat equation with initial value $u_{0}$, then the periods of $u(\cdot, t)$ are constant in $t$ and $\lim _{t \rightarrow \infty} u(\cdot, t)$ is the harmonic form cohomologous to $u_{0}$. More generally, let $H$ denote orthogonal projection onto the harmonic $p$ forms. Then for any smooth $p$ form $f$ on $M$,

$$
H f=\lim _{t \rightarrow \infty} e^{-t \Delta} f
$$

The heat operator can be used to construct the Green's operator, or fundamental solution, $G$ of the inhomogeneous Laplace equation. Let

$$
G=\int_{0}^{\infty}\left(e^{-t \Delta}-H\right) d t
$$

Then $u=G f$ solves

$$
\Delta u=f-H f .
$$

Another type of application of the heat operator is given by Minakshisundaram and Pleijel [MP]. Let $0 \leqslant \lambda_{1} \leqslant \lambda_{2} \leqslant \cdots$ be the spectrum of the Laplacian on functions. Then

$$
\operatorname{tr} e^{-t \Delta}=\sum e^{-t \lambda}
$$


which converges for $t>0$. Also

$$
\operatorname{tr} e^{-t \Delta}=\int_{M} p(x, x, t) d V(x)
$$

where $d V$ is the volume element on $M$, so $p(x, x, t)$ is a local form of the trace. Minakshisundaram [Min] proved the existence of an asymptotic expansion for $p(x, x, t)$ as $t \rightarrow 0$,

$$
p(x, x, t) \sim\left(\frac{1}{4 \pi t}\right)^{n / 2} \sum_{j \geqslant 0} U_{j}(x) t^{J}
$$

and $U_{0} \equiv 1$. Hence there is an asymptotic expansion for the trace of the heat operator on functions,

$$
\sum e^{-t \Delta} \sim\left(\frac{1}{4 \pi t}\right)^{n / 2} \sum_{j \geqslant 0} c_{j} t^{j}
$$

and $c_{0}$ is the volume of $M$. Thus, the spectrum of the Laplacian on functions determines the dimension and volume of $M$.

Gaffney [Ga] generalized (1.9) and (1.10) to forms. Later, McKean and Singer [MS] extended them to forms satisfying Dirichlet or Neumann boundary conditions on compact oriented Riemannian manifolds with boundary. The leading term of the asymptotic expansion on manifolds with boundary is the same as for manifolds without boundary. This generalizes work of Carleman [Ca] and Pleijel [PI 1, PI 2] on domains in $\mathbf{R}^{2}$ and $\mathbf{R}^{3}$. Greiner [Gr] and Seeley [Se 1, Se 2] extended the expansions to general elliptic operators and elliptic boundary value problems.

The oldest result in spectral geometry is Weyl's Theorem.

THEOREM (WEYL [Wey]). Let $\Omega$ be a bounded domain in $\mathbf{R}^{2}$ with smooth boundary. Let $0<\lambda_{0} \leqslant \lambda_{1} \leqslant \lambda_{2} \leqslant \cdots$ be the eigenvalues of the Laplacian on functions satisfying Dirichlet boundary conditions (i.e. vanishing on $\partial \Omega$ ). Then

$$
\lim _{n \rightarrow \infty} \frac{n}{\lambda_{n}^{1 / 2}}=\frac{A}{4 \pi}
$$

where $A$ is the area of $\Omega$.

By Karamata's Tauberian Theorem, this result is equivalent to the fact that if $n=2$, the first term $c_{0}$ in the asymptotic expansion (1.10) is the area. Dodziuk [Dod] has a more detailed discussion of the heat equation and Weyl's theorem for domains in $\mathbf{R}^{n}$.

There is a famous problem, the title of Kac's article [Ka] and film, "Can one hear the shape of a drum?" (This title was suggested by Bers.) To what extent does the spectrum determine the manifold? It determines the dimension and volume. However, Milnor [Mil] gave an example of two sixteen-dimensional flat tori which are not isometric but have the same spectrum, the first example of isospectral, nonisometric manifolds. Here, by isospectral I mean having the same spectrum on functions. Vigneras [V] gave examples of isospectral, nonisometric compact Riemann surfaces. These surfaces are diffeomorphic because $c_{1}$ 
is the total curvature [MS]. She also gave examples of isospectral, nonhomeomorphic compact three manifolds. Vigneras' examples all have constant negative curvature. Ikeda [I] gave examples of isospectral, nonisometric lens spaces of dimension 5 (and some larger dimensions). These spaces are not homeomorphic and, in some cases, not homotopy equivalent. Urakawa [U] gave examples of isospectral, nonisometric bounded domains in $\mathbf{R}^{n}, n \geqslant 4$, with piecewise smooth boundary. These domains are topologically balls. All the examples I have mentioned so far are discrete. Gordon and Wilson [GW] have shown the existence of nontrivial isospectral deformations of certain compact nilmanifolds and solvmanifolds. Thus, there are now many examples which show that the spectrum does not determine the geometry.

However, there is much geometric information in the spectrum. I will describe a few of these results. McKean and Singer [MS] proved that the coefficients $U_{j}(x)$ in the Minakshisundaram-Pleijel expansion (1.9), and more generally in the analogous expansion for $p$ forms, are universal polynomials (depending only on the dimension, $j$ and $p$ ) in the curvature and its covariant derivatives. They also showed that the Euler characteristic is the alternating sum over $p$ of the coefficients of $t^{0}$ in the expansion (1.10) on $p$ forms. By calculating certain combinations of the coefficients of $t^{j}, j \leqslant 0$, on all degrees of forms, Patodi [Pa 2] proved that the spectra on $p$ forms for all $p$ determines the Chern-Gauss-Bonnet integrand, and hence obtained a new proof of the Chern-Gauss-Bonnet Theorem. By calculating the first three coefficients on functions, one forms and two forms, he showed [Pa 1] that the spectra determine whether $M$ is flat, has constant scalar curvature, has constant sectional curvature (hence is a sphere with the standard metric), or is an Einstein space. The idea of the proof is that the coefficients determine a certain quantity whose integral over $M$ is zero if and only if $M$ has the geometric property one is considering.

The Chern-Gauss-Bonnet Theorem is probably the simplest special case of the Atiyah-Singer Index Theorem. Patodi [Pa 3] also proved another special case, the Hirzebruch-Riemann-Roch Theorem for Kähler manifolds, by similar methods. Atiyah, Bott and Patodi [ABP] used heat equation methods and Gilkey's invariant theory [Gi 1] to prove the Index Theorem.

The proofs of these geometric results depend on local invariants, that is, invariants which are obtained by integrating differential forms over the manifold. However, the spectrum of the Laplacian on $p$ forms also determines some nonlocal invariants. The simplest nonlocal invariant is the $p$ th Betti number. Ray and Singer [RS 1] introduced a nonlocal invariant, the analytic torsion. Let $\chi$ be a character of $\pi_{1}(M)$, and $L(\chi)$ the associated flat line bundle. Suppose $L(\chi)$ is acyclic, i.e. there are no $L(\chi)$-valued harmonic $p$ forms for any $p$. Define the $p$ th zeta function by

$$
\zeta_{p}(s, \chi)=\sum \lambda^{-s},
$$

where the sum is taken over all eigenvalues of the Laplacian on $p$ forms with values in $L(\chi)$. Then $\zeta_{p}(s, \chi)$ is holomorphic for $\operatorname{Re} s>n / 2$, has a meromorphic continuation to the plane, and is regular at $s=0$. This follows from 
looking at the corresponding heat operator, since $\zeta_{p}(s, \chi)$ is the Mellin transform of $\operatorname{tr} \exp \left(-t \Delta_{p}^{x}\right)$,

$$
\zeta_{p}(s, \chi)=\frac{1}{\Gamma(s)} \int_{0}^{\infty} t^{s-1} \operatorname{tr} \exp \left(-t \Delta_{p}^{x}\right) d t
$$

where $\Delta_{p}^{\chi}$ denotes the Laplacian on $L(\chi)$-valued $p$ forms. For $\operatorname{Re} s>n / 2$,

$$
\zeta_{p}^{\prime}(s, \chi)=-\sum(\log \lambda) \lambda^{-s},
$$

so $\zeta_{p}^{\prime}(0, \chi)$ gives a way of making sense of the logarithm of the "determinant", and hence the "determinant" of $\Delta_{p}^{\chi}$. The analytic torsion $T(M, \chi)$ is defined by

$$
\log T(M, \chi)=\frac{1}{2} \sum_{p=0}^{n}(-1)^{p} \zeta_{p}^{\prime}(0, \chi) .
$$

This nonlocal invariant is independent of the metric. Ray and Singer conjectured that it was equal to the Reidemeister torsion, and Ray [Ray] verified it for lens spaces. The conjecture was later proved by Cheeger [Ch 1] and Müller [Mu]. The special case of lens spaces shows that by considering the spectrum of $\Delta_{p}^{\chi}$ for all $p$ and $\chi$, one can tell that Ikeda's examples are nonisometric.

In addition to giving rise to geometric invariants, the heat kernel has proved to be a useful tool for obtaining other results. For examples, Cheng and $\mathrm{Li}$ [CL] used estimates for the heat kernel to obtain geometric lower bounds for the eigenvalues $\lambda_{k}$ of the Laplacian on functions. Li and Yau [LY] extended this method to study the Schrödinger equation.

Cheeger [Ch 2] has extended many results on the heat kernel to spaces with cone-like singularities. In particular, he obtains an asymptotic expansion and uses this to calculate the $L^{2}$ Euler characteristic and the signature.

2. The heat equation in Hermitian geometry. Let $M$ be a compact Hermitian manifold of complex dimension $n$. A $(p, q)$ form on $M$ is a complex valued $p+q$ form which involves $p d z$ 's and $q d \bar{z}$ 's, where $z$ is a local holomorphic coordinate map on $M$. The $\bar{\partial}$-Laplacian on $(p, q)$ forms is

$$
\Delta_{p, q}=\bar{\partial} \bar{\partial} *+\bar{\partial} * \bar{\partial} .
$$

Because $\Delta_{p, q}$ is elliptic, the fundamental solution of the corresponding heat equation has a smooth kernel $P_{p, q}(z, w, t)$. The local trace $P_{p, q}(z, z, t)$ again has an asymptotic expansion

$$
P_{p, q}(z, z, t) \sim \sum_{j \geqslant-n} c_{j}(z) t^{j} .
$$

Patodi's proof of the Hirzebruch-Riemann-Roch Theorem on Kähler manifolds [ $\mathrm{Pa} \mathrm{3}$ ] came from calculating certain combinations of the coefficients of $t^{j}$ for $j \leqslant 0$ on $(0, q)$ forms, $0 \leqslant q \leqslant n$. Gilkey [Gi 2], by calculating the second coefficient on functions, $(1,0)$ and $(0,1)$ forms, showed that the spectra on these degrees of forms determines whether $M$ is Kähler. Donnelly [Don] and Gilkey-Sacks [Gi Sa] showed that it determines whether $M$ is $\mathbf{C} P^{n}$ with the standard metric. These geometric results all rely on local invariants. 
The simplest nonlocal spectral invariant on complex manifolds is the $(p, q)$ Betti number, or Hodge number, the dimension of the kernel of $\Delta_{p, q}$. Ray and Singer [RS 2] introduced a nonlocal invariant, the holomorphic torsion, which is the complex analogue of the analytic torsion. Let $\chi: \pi_{1}(M) \rightarrow S^{1}$ be a character of the fundamental group of $M$ and $L(\chi)$ the associated flat holomorphic line bundle. Assume that for some $p$, the $\bar{\partial}$-cohomology on $(p, q)$ forms with values in $L(\chi)$ vanishes for all $q$. Let $\Delta_{p, q}^{\chi}$ denote the $\bar{\partial}$-Laplacian on $(p, q)$ forms with values in $L(\chi)$. Define the $(p, q)$ zeta function by

$$
\zeta_{p, q}(s, \chi)=\sum \lambda^{-s}
$$

where the sum is over all eigenvalues of $\Delta_{p, q}^{x}$. Then this series converges for $\operatorname{Re} s>n$, and $\zeta_{p, q}(s, \chi)$ is the Mellin transform of $\operatorname{tr} \exp \left(-t \Delta_{p, q}^{\chi}\right)$, hence has a meromorphic extension to the plane which is regular at $s=0$. As in (1.12), $\zeta_{p, q}^{\prime}(0, \chi)$ gives a way of making sense of the logarithm of the "determinant" of $\Delta_{p, q}$. The holomorphic torsion $T_{p}(M, \chi)$ is defined by

$$
\log T_{p}(M, \chi)=\frac{1}{2} \sum_{q=0}^{n}(-1)^{q} q \zeta_{p, q}^{\prime}(0, \chi) .
$$

Now, $T_{p}(M, \chi)$ depends on the complex structure on $M$, the Hermitian metric and the character $\chi$. If $\chi^{\prime}$ is another such character, then the ratio

$$
T_{p}(M, \chi) / T_{p}\left(M, \chi^{\prime}\right)
$$

is independent of the choice of metric, hence gives an invariant of the complex structure. Ray and Singer hoped that this invariant would prove useful in distinguishing complex structures where other methods, for example the period mapping, do not apply. As far as I know, this is still an open problem. The torsion has been calculated in a number of examples [RS 2, St 1], including Riemann surfaces, Hopf manifolds and compact even-dimensional Lie groups. In these examples, the answer is in terms of certain functions which arise in analytic number theory. These examples show that the holomorphic torsion is not equal to any previously known invariant. However, in these examples there are many other methods available for distinguishing complex structures.

Fay [F] showed that the holomorphic torsion determines the complex structure of a Riemann surface. For a Riemann surface $M$ it turns out [RS 2] that

$$
\log T_{p}(M, \chi)=-\frac{1}{2} \zeta_{0,0}^{\prime}(0, \chi)
$$

Hence, in Vigneras' examples of isospectral, nonisometric Riemann surfaces, if, instead of just considering the spectrum of the Laplacian on functions, one considers it on sections of $L(\chi)$ for each character $\chi$ of the fundamental group of $M$, one can tell that the surfaces are not isometric.

Let $E$ be a holomorphic, Hermitian vector bundle over a compact complex manifold, and let $\Delta_{p, q}^{E}$ denote the $\bar{\partial}$-Laplacian on $E$-valued $(p, q)$ forms. Again, form a zeta function

$$
\zeta_{p, q}(s, E)=\sum \lambda^{-s}
$$


where the sum is over all positive eigenvalues of $\Delta_{p, q}^{E}$. This has the same analyticity properties as the other zeta functions I have considered. Define

$$
\operatorname{Det}_{\zeta} \Delta_{p, q}^{E}=\exp \left(-\zeta_{p, q}^{\prime}(0, E)\right) .
$$

For Riemann surfaces, $\left(\operatorname{Det}_{\zeta} \Delta_{0,1}^{E}\right)^{-1}$ is a generalization of $T_{0}^{2}$. Also

$$
\operatorname{Det}_{\zeta} \Delta_{0,1}^{E}=\operatorname{Det}_{\zeta} \Delta_{0,0}^{E},
$$

a generalization of (2.6). Quillen [Q] has used $\operatorname{Det}_{\zeta} \Delta_{0,0}^{E}$ to define a metric on the determinant line of a holomorphic Hermitian vector bundle $E$ over a Riemann surface which is smooth on holomorphic families of bundles and invariant under gauge transformations.

"Determinants" of elliptic operators and analytic and holomorphic torsion also arise in theoretical physics, for example, in studying dual resonance models [Ma] and in studying the partition function of certain quadratic functionals [Sch].

3. The heat equation for the $\bar{\partial}_{b}$-Laplacian. Let $\Omega$ be a domain in $\mathbf{C}^{n+1}, n \geqslant 1$, with $C^{\infty}$ boundary $M$. Let $T^{1,0}(M)$ denote the linear combinations of the vector fields $\partial / \partial z^{1}, \ldots, \partial / \partial z^{n+1}$ which are in the complexified tangent bundle of $M$. Let $T^{0,1}(M)=T^{1,0}(M)$. Let $r$ be a defining function for $\Omega$ with $\Omega=\{r<0\}, M=\{r=0\}$ and $d r \neq 0$ on $M$.

Definition 3.1. The domain $\Omega$ is strictly pseudoconvex if the Hermitian form $L$, defined on $T^{1,0}(M)$ by

$$
L(X, Y)=\frac{1}{2} \partial \bar{\partial} r(X, \bar{Y})
$$

is positive definite. The form $L$ is called the Levi form of $r$.

This definition is independent of the choice of $r$ because $\bar{\partial} r$ vanishes on $T^{1,0}(M)$. Strict pseudoconvexity is equivalent to a geometric condition. The domain $\Omega$ is strictly pseudoconvex if and only if it is locally biholomorphic to a strictly convex domain by a biholomorphic map smooth up to the boundary [Kr]. By strictly convex I mean a domain having a defining function whose Hessian is positive definite. In particular, any strictly convex domain with smooth boundary, for example, an ellipsoid, is strictly pseudoconvex. If $\Omega=B$, the unit ball in $\mathbf{C}^{n+1}$, we may take $r=|z|^{2}-1$. Then the Levi form of $r$ is the standard Euclidean inner product. Another example of a strictly pseudoconvex domain is the Siegel domain

$$
D=\left\{(z, w): z \in \mathbf{C}^{n}, w \in \mathbf{C}, \operatorname{Im} w>|z|^{2}\right\} .
$$

The vector fields

$$
Z_{j}^{H}=\frac{\partial}{\partial z^{j}}+2 i \bar{z}^{j} \frac{\partial}{\partial w}, \quad j=1, \ldots, n,
$$

form a basis for $T^{1,0}(\partial D)$, and the Levi form of

$$
\rho(z, w)=|z|^{2}-\operatorname{Im} w
$$


is positive definite. In fact, $D$ is biholomorphically equivalent to the ball by the linear fractional transformation

$$
(z, w) \rightarrow\left(\frac{2 z}{w+i}, \frac{w-i}{w+i}\right)
$$

and this map extends smoothly to $\bar{D}$.

We fix a defining function $r$ as above for $\Omega$.

Definition 3.2. A Levi metric determined by $r$ on $M$ is a Hermitian inner product $\langle$,$\rangle on T(M) \otimes \mathbf{C}$ such that

(i) If $X, Y \in T^{1,0}(M, p),\langle X, Y\rangle=L(X, Y)$.

(ii) $T^{1,0}(M) \perp T^{0,1}(M)$ and conjugation commutes with the metric.

(iii) $\langle i \bar{\partial} r, i \bar{\partial} r\rangle \equiv 1$.

A Levi metric determines a unique real transversal vector field $T \perp T^{1,0}(M)$ satisfying $i \bar{\partial} r(T) \equiv 1$. A Levi metric is distinguished if $T\lrcorner \partial \bar{\partial} r \equiv 0$.

A defining function $r$ determines a unique distinguished Levi metric. If $M=S^{2 n+1}$ and $r=|z|^{2}-1$, this is just the standard metric. If $M=\partial D$, the boundary of the Siegel domain and $\rho$ is given by (3.3), then $\left\{Z_{1}^{H}, \ldots, Z_{n}^{H}\right.$, $\left.\bar{Z}_{1}^{H}, \ldots, \bar{Z}_{n}^{H}, T^{H}\right\}$ is an orthonormal basis for the distinguished Levi metric determined by $\rho$, where $w=u+i v$ and $T^{H}=\partial / \partial u$.

Let $\Lambda^{0,1}(M)$ denote the space of complex one forms on $M$ which annihilate $T^{1,0}(M)$ and the transversal vector field $T$, and let $\Lambda^{0, q}(M)$ denote the $q$ th exterior power of $\Lambda^{0,1}(M)$. Then the $\bar{\partial}$-boundary operator $\bar{\partial}_{b}: C^{\infty}\left(\Lambda^{0, q}(M)\right) \rightarrow$ $C^{\infty}\left(\Lambda^{0, q+1}(M)\right)$ is defined by $\bar{\partial}_{b}=\pi_{0, q+1} \circ d$, where $d$ is exterior derivative and $\pi_{0, q}$ is orthogonal projection onto $\Lambda^{,, q}$. (By working with a quotient bundle of $\Lambda^{q}$ instead of a subbundle, one can give an intrinsic definition of $\bar{\partial}_{b}$. I have not done that because I need the metric for the next step.) The $\bar{\partial}_{b}$-Laplacian $\square_{b}$ is defined by $\square_{b}=\bar{\partial}_{b} \bar{\partial}_{b}^{*}+\bar{\partial}_{b}^{*} \bar{\partial}_{b}$, where $\bar{\partial}_{b}^{*}$ is the formal adjoint of $\bar{\partial}_{b}$. For simplicity I will just consider $\square_{b}$ on $C^{\infty}\left(\Lambda^{0,1}(M)\right)$. Let $\left\{Z_{1}, \ldots, Z_{n}\right\}$ be a local orthonormal basis of $T^{1,0}(M)$, and $\left\{\omega^{1}, \ldots, \omega^{n}\right\}$ the dual basis of $\Lambda^{1,0}(M)$ $=\overline{\Lambda^{0,1}(M)}$. If $\varphi \in C^{\infty}\left(\Lambda^{0,1}(M)\right)$, then locally, $\varphi=\sum \varphi_{j} \bar{\omega}^{j}$. A straightforward calculation shows that

$$
\square_{b}\left(\sum \varphi_{j} \bar{\omega}^{j}\right)=\sum\left(\left(-\frac{1}{2} \sum_{k=1}^{n}\left(Z_{k} \bar{Z}_{k}+\bar{Z}_{k} Z_{k}\right)+i(n-2) T\right) \varphi_{j}\right) \bar{\omega}^{j}+L \varphi,
$$

where $L$ is a first order differential operator involving only the $Z_{j}$ and $\bar{Z}_{j}$, not $T$. If $M=\partial D$ and $\left\{\omega_{H}^{j}\right\}$ is the dual basis to $\left\{Z_{j}^{H}\right\}$, then the operator $L$ of (3.5) is zero, so $\square_{b}^{H}$ is the diagonal operator $\mathscr{L}_{n-2}$ where

$$
\mathscr{L}_{n-2}=-\frac{1}{2} \sum_{k=1}^{n}\left(Z_{k}^{H} \bar{Z}_{k}^{H}+\bar{Z}_{k}^{H} Z_{k}^{H}\right)+i(n-2) T^{H} .
$$

By (3.5), $\square_{b}$ is not elliptic; it does not involve second order derivatives in $T$. As a result, the term $i(n-2) T$ is as important as the second order terms, but $L$ is not. In fact, modulo $T^{1,0}(M) \oplus T^{0,1}(M), T$ is $(i / 2)\left[Z_{k}, \bar{Z}_{k}\right]$.

From now on, I will assume $M$ is compact and $n \geqslant 2$. A form $\varphi(\cdot, t) \in$ $\Lambda^{0,1}(M), t \in \mathbf{R}^{+}$, solves the heat equation for $\square_{b}$ if

$$
\left(\partial / \partial t+\square_{b}\right) \varphi=0 .
$$


The initial value problem is: given $\varphi_{0} \in C\left(\Lambda^{0,1}(M)\right)$, find a solution $\varphi(\cdot, t)$ of the heat equation (3.7) with

$$
\lim _{t \rightarrow 0} \varphi(\cdot, t)=\varphi_{0}
$$

By Kohn's subelliptic estimates and regularity results for $\square_{b}[\mathbf{F K}]$, there is a complete orthonormal set $\left\{\varphi_{i}\right\}$ of eigenforms for $\square_{b}$ with corresponding eigenvalues $\left\{\lambda_{i}\right\}$. Let

$$
p(x, y, t)=\sum e^{-\lambda_{i} t} \varphi_{i}(x) \otimes \overline{\varphi_{i}(y)} .
$$

Then, using Kohn's results, one sees [St 2] that $p$ is $C^{\infty}$ on $M \times M \times \mathbf{R}^{+}$and is the unique fundamental solution of the initial value problem for the heat equation for $\square_{b}$,

$$
\varphi(x, t)=\int_{M} p(x, y, t) \wedge * \varphi_{0}(y) .
$$

To obtain analogues of the results in Riemannian and Hermitian geometry, one needs much more explicit information about the heat kernel than (3.9) - analogues of the explicit construction of the kernel and of the asymptotic expansion. The first step is to have an analogue of the Gaussian-because this heat equation is degenerate parabolic, it cannot be modeled on the Euclidean heat equation. However, formulas (3.5) and (3.6) suggest the heat equation for $\mathscr{L}_{n-2}$ on $\partial D$ as a model. This should be a good model because $\partial D$ is a particularly simple boundary; it can be identified with the Heisenberg group $H_{n}$ with group law

$$
(z, w)\left(z^{\prime}, w^{\prime}\right)=\left(z+z^{\prime}, w+w^{\prime}+2 i \sum_{j=1}^{n} \bar{z}^{j} z^{\prime j}\right),
$$

and $\mathscr{L}_{n-2}$ is left invariant, as are the vector fields $Z_{j}^{H}$ and $T^{H}$. Thus one can exploit the group structure. Of course, $H_{n}$ is not compact, so for the heat equation I require the initial data to be compactly supported and the solution to be square integrable for each $t$. Then the fundamental solution $e^{-t \mathscr{L}_{n-2}}$ is the operator given by convolution with respect to the volume element with

$$
r_{t}^{n-2}(z, u)=\left(\frac{1}{2 \pi t}\right)^{n+1} \int_{-\infty}^{\infty}\left(\frac{\tau}{\sinh \tau}\right)^{n} \exp \left(-i \tau \frac{u}{t}-\frac{|z|^{2} \tau}{t \tanh \tau}-(n-2) \tau\right) d \tau
$$

where $u=\operatorname{Re} w$ so $(z, u)$ give coordinates on $\partial D=H_{n}$ [St 3, Theorem I 4.9].

I will describe two approaches to studying $p(x, y, t)$. The first, due to Stanton and Tartakoff [ST] is the iterative construction of the fundamental solution. This approach gives the exact solution of the heat equation. Previous work on $\square_{b}$ on general boundaries of strictly pseudoconvex domains has only led to parametrices for the problems under consideration. Our approach is motivated by the McKean-Singer [MS] construction of the fundamental solution in Riemannian geometry and the Folland-Stein [FS] construction of a parametrix for $\square_{b}$. Folland and Stein observed that one can find an osculating Heisenberg group, that is, one can find coordinates on $M$ which make precise 
the statement that $\square_{b}$ looks locally very much like the diagonal operator $\mathscr{L}_{n-2} \cdot I$, where $I$ is the identity (see also [Gr St]). As before, let $\left\{Z_{1}, \ldots, Z_{n}\right\}$ be an orthonormal basis of $T^{1,0}(M)$ over an open set $U$. There is a map $\Theta$ : $U \times U \rightarrow H_{n}$, called an admissible coordinate map such that for $x \in U$, $\Theta(x, x)=0, \Theta(x, \cdot)$ is a coordinate map, and in these coordinates

$$
Z_{j}=Z_{j}^{H}+\sum_{k}\left(\mathcal{O}^{1} Z_{k}+\mathcal{O}^{1} \bar{Z}_{k}\right)+\mathcal{O}^{2} T, \quad T=T^{H}+\mathcal{O}^{1} .
$$

Here, a function $f \in \mathcal{O}^{1}$ if $f(x)=0$ and $f \in \mathcal{O}^{2}$ if $f \in \mathcal{O}^{1}$ and $\left(Z_{j} f\right)(x)=$ $\left(\bar{Z}_{j} f(x)\right)=0, j=1, \ldots, n$. The map $\Theta$ is not unique. The error terms in the first equality of (3.13) are "morally zeroth order", and in the second equality "morally first order", hence,

$$
\square_{b}=\mathscr{L}_{n-2} I+L^{\prime},
$$

where $L^{\prime}$ is a second order operator which is "morally first order". We call the coordinates $\Theta(x, \cdot)$ admissible coordinates centered at $x$.

Now cover $M$ by open sets $U_{i}, i=1, \ldots, m$, and choose an orthonormal basis $\left\{Z_{j}^{i}\right\}_{j=1}^{n}$ of $T^{1,0}\left(U_{i}\right)$ with dual basis $\left\{\omega_{i}^{i}\right\}_{j=1}^{n}$ of $\Lambda^{1,0}\left(U_{l}\right)$. Let $\Theta_{i}: U_{i} \times U_{i}$ $\rightarrow H_{n}$ be an admissible coordinate map and $\left\{\varphi_{i}^{2}\right\}$ a partition of unity subordinate to $\left\{U_{i}\right\}$. Let

$$
r(x, y, t)=\sum_{i=1}^{m} \sum_{j=1}^{n} \varphi_{i}(x) r_{t}^{n-2}\left(\Theta_{i}(y, x)\right) \varphi_{i}(y) \bar{\omega}_{i}^{j}(x) \otimes \omega_{i}^{j}(y) .
$$

Then near the diagonal, $r$ looks like $r_{t}^{n-2} \cdot I$. This is our analogue of the "Gaussian" (1.3); to first approximation $p(x, y, t) \sim r(x, y, t)$. Let $q(x, y, t)$ $=\left(\square_{b}+\partial / \partial t\right) r(x, y, t)$. Then $q$ is a measure of the error in the approximation. If $f$ is a section of $\Lambda^{0,1} \otimes \Lambda^{1,0}$ over $M \times M \times \mathbf{R}^{+}$and $g$ is a section of $\Lambda^{0,1}$ over $M \times \mathbf{R}^{+}$(possibly also depending on a parameter $\zeta$ ), define a new section $f \# g$ of $\Lambda^{0,1}$ over $M \times \mathbf{R}^{+}$by

$$
(f \# g)(x, \zeta, t)=\int_{0}^{t} \int_{M} f(x, z, t-s) \wedge * g(z, \zeta, s) d s,
$$

assuming the integral is defined. The key properties of $r$ are contained in the following.

Proposition 3.1. (i) $r$ is $C^{\infty}$.

(ii) If $f \in C\left(\Lambda^{1,0}(M)\right)$,

$$
\lim _{t \rightarrow 0} \int_{M} r(x, y, t) \wedge * f(y)=f(x) .
$$

(iii) There is a constant $c$ such that

$$
\int_{M}|r(x, y, t)| d V \leqslant c,
$$

where $d V$ is the volume element in either variable. 
(iv) For each $T>0$, there is a constant $c$ such that for $t \leqslant T$,

$$
|q(x, y, t)| \leqslant \frac{c}{t^{n+3 / 2}}, \quad \int_{M}|q(x, y, t)| d V \leqslant \frac{c}{\sqrt{t}} .
$$

(v) If $f$ is a sufficiently smooth section of $\Lambda^{0,1}$ over $M \times \mathbf{R}^{+}$,

$$
\left(\square_{b}+\partial / \partial t\right) r \# f=f+q \# f .
$$

Property (i) is immediate from the definition, and (ii)-(iii) follow from the corresponding properties of $r_{t}^{n-2}$. Because $r_{t}^{n-2}$ solves the heat equation for $\mathscr{L}_{n-2}$, (iv) follows from (3.14). This property says that $r$ is a good first approximation; the worst singularities of the individual terms cancel. The last property follows formally from differentiating under the integral and using (ii). For sufficiently smooth $f$, this formal argument can be justified. This property says that $r$ gives a good first approximation to the fundamental solution of the inhomogeneous heat equation, since $q \# f \rightarrow 0$ as $t \rightarrow 0$.

Let $q^{1}=q$ and $q^{k}=q \# q^{k-1}, k \geqslant 2$.

THEOREM 3.2 [ST]. The fundamental solution $p(x, y, t)$ of the heat equation for $\square_{b}$ is

$$
p(x, y, t)=r(x, y, t)+\sum_{k=1}^{\infty}(-1)^{k}\left(r \# q^{k}\right)(x, y, t) .
$$

Furthermore,

$$
p-r=\mathcal{O}\left(t^{-n-1 / 2}\right) \text { as } t \rightarrow 0,
$$

and

$$
\int_{M}|(p-r)(x, y, t)| d V(y)=\mathcal{O}(\sqrt{t}) \text { as } t \rightarrow 0 .
$$

Formally, by Proposition 3.1(v), the right side of (3.17) satisfies the heat equation. By Proposition 3.1(ii) and (3.19) it reproduces initial values. Formula (3.17) is formally like the McKean-Singer formula for the fundamental solution of the heat equation in Riemannian geometry [MS]. For parabolic problems such series solutions are classical, going back to E. E. Levi. However, because of the nonellipticity of $\square_{b}$, classical methods do not apply to estimating the terms and proving the convergence in (3.17). Thus, the hard work is to find the appropriate estimates.

Corollary 3.3 (Weyl TYPe TheORem). Let $N(\lambda)$ denote the number of eigenvalues (with multiplicity) of $\square_{b}$ which are less than $\lambda$. Then

$$
N(\lambda) \sim \frac{n}{(2 \pi)^{n+1}}\left(\int_{-\infty}^{\infty}\left(\frac{\tau}{\sinh \tau}\right)^{n} e^{-(n-2) \tau} d \tau\right) \frac{\operatorname{Vol} M}{\Gamma(n+2)} \lambda^{n+1}
$$

as $\lambda \rightarrow \infty$.

The function $N(\lambda)$ grows at a rate $\lambda^{1 / 2}$ greater than the corresponding function for an elliptic problem would. The corollary follows from (3.18), the formula for $r$ (3.15), and Karamata's Tauberian Theorem. 
The second approach to constructing the fundamental solution of the heat equation for $\square_{b}$ is due to Beals, Greiner and Stanton [BGS]. Our approach is motivated by the Greiner [Gr] and Seeley [Se 1] constructions of parametrices for the heat equation in Riemannian geometry together with the Beals-Greiner [BG] pseudodifferential operator calculus for a class of operators which includes $\square_{b}$ and a parametrix for $\square_{b}$. We use an appropriate modification of this calculus to construct a parametrix $Q$ for the problem. Our $Q$ differs from the fundamental solution $P$ by a smoothing operator, and thus its kernel contains all the information about the asymptotic behavior of $p$ as $t \rightarrow 0$.

We want a class of pseudodifferential operators for which there is a symbol calculus and which includes a parametrix for $\partial / \partial t+\square_{b}$, as well as $\partial / \partial t+\square_{b}$. This class should take into account the natural homogeneity of the problem, so $Z_{j}, \bar{Z}_{j}$ should be considered to be operators of order 1 , and $\partial / \partial t$ and $T$ should be considered of order 2 . This reflects the fact that $T \equiv(i / 2)\left[Z_{k}, \bar{Z}_{k}\right] \bmod T^{1,0}$ $\oplus T^{0,1}$, and makes $\partial / \partial t+\mathscr{L}_{n-2}$ homogeneous of order 2 . Treating $\partial / \partial t$ as an operator of order 2 in parabolic equations is classical [Gr]. The Beals-Greiner [BG] pseudodifferential operator calculus is designed to treat $T$ that way. This is the natural homogeneity on the Heisenberg group, and the group will again come into constructing the parametrix.

We build our calculus from an appropriate class of homogeneous functions.

Definition 3.3. For $m \in \mathbf{Z}, \mathscr{F}_{m, h}$ is the subspace of $C^{\infty}\left(\left(\mathbf{R}^{2 n+1} \times \overline{\mathbf{C}}_{-}\right) \backslash 0\right)$ consisting of functions $f$ which are holomorphic in the last variable and homogeneous of degree $m$ with respect to the dilations

$$
\lambda(\sigma, \tau)=\left(\lambda^{2} \sigma^{0}, \lambda \sigma^{\prime}, \lambda^{2} \tau\right),
$$

$\lambda \in \mathbf{R} \backslash 0, \sigma=\left(\sigma^{0}, \sigma^{\prime}\right) \in \mathbf{R}^{2 n+1}$, and $\tau \in \overline{\mathbf{C}}_{-}$.

To use these to build pseudodifferential operators on $M \times \mathbf{R}$, we localize. Let $U$ be a coordinate patch on $M$ with coordinate $x$. Fix an orthonormal basis $\left\{Z_{j}\right\}$ of $T^{1,0}(M)$. Let $X_{j}=2 \operatorname{Re} Z_{j}, X_{j+n}=-2 \operatorname{Im} Z_{j}, j=1, \ldots, n$, and $X_{0}=T$. Then, in terms of the local coordinates $x$ and dual coordinates $\xi$ on $T^{*} U$, let $\sigma^{j}(x, \xi)$ be the symbol of $(1 / i) X_{j}$ and $\tau$ the symbol of $(1 / i)(\partial / \partial t)$. For our homogeneous symbols of order $m$ on $U$ we take the following.

Definition 3.4. For $m \in \mathbf{Z}, S_{m, h}(U \times \mathbf{R})$ is the subspace of $n \times n$ matrices $q$ of functions $q_{i j}, q_{i j} \in C^{\infty}\left(U \times\left[\left(\mathbf{R}^{2 n+1} \times \overline{\mathbf{C}}_{-}\right) \backslash 0\right]\right)$, of the form

$$
q_{i j}(x, \xi, \tau)=f(x, \sigma(x, \xi), \tau),
$$

where for fixed $x, f_{x}(\sigma, \tau)=f(x, \sigma, \tau) \in \mathscr{F}_{m, h}$.

Thus, $\sigma^{0} I$ and $\tau I \in S_{2, h}(U \times \mathbf{R})$ and $\sigma^{j} I \in S_{1, h}(U \times \mathbf{R})$ for $j \geqslant 1$, so $X_{0} I$ and $(\partial / \partial t) I$ are second order and $X_{j} I, j \geqslant 1$, are first order for our calculus.

Definition 3.5. For $m \in \mathbf{Z}, S_{h}^{m}(U \times \mathbf{R})$ is the subspace of matrices $q$ of functions in $C^{\infty}\left(U \times \mathbf{R}^{2 n+2}\right)$ which have an asymptotic expansion

$$
q \sim \sum_{j=0}^{\infty} q_{m-j}, \quad q_{m-j} \in S_{m-j, h}(U \times \mathbf{R}) .
$$

If $q_{m} \neq 0$, we call $q_{m}$ the principal symbol of $q$. 
The symbol of $\partial / \partial t+\square_{b}$ is in $S_{h}^{2}(U \times \mathbf{R})$. Its "principal part" $\partial / \partial t+$ $\left(\square_{b}-L\right)$ (see (3.5)) has symbol

$$
\left(i \tau+\frac{1}{4}\left|\sigma^{\prime}(x, \xi)\right|^{2}-(n-2) \sigma^{0}(x, \xi)\right) I \in S_{2, h}(U \times \mathbf{R}),
$$

and $L$ has a symbol in $S_{h}^{1}(U \times \mathbf{R})$.

If $q \in S_{h}^{m}(U \times \mathbf{R})$, it is the symbol of a pseudodifferential operator $Q$ on $U \times \mathbf{R}$,

$$
(Q u)(x, t)=(2 \pi)^{-2 n-2} \int e^{i\langle(x, t)(\xi, \tau)\rangle} q(x, \sigma(x, \xi), \tau) \hat{u}(\xi, \tau) d \xi d \tau
$$

for $u \in C_{0}^{\infty}(U \times \mathbf{R})$. This class of operators is independent of the choices of coordinates and vector fields $X_{j}$. Hence, $\mathrm{Op}\left(S_{h}^{m}(M \times \mathbf{R})\right)$, the class of pseudodifferential operators which when localized have symbols in $S_{h}^{m}(U \times \mathbf{R})$, is well defined.

Following the methods of [BG], we have proved that if

$$
Q_{i} \in \mathrm{Op}\left(S_{h}^{m_{i}}(M \times \mathbf{R})\right), \quad i=1,2,
$$

then

$$
Q=Q_{1} \circ Q_{2} \in \operatorname{Op}\left(S_{h}^{m_{1}+m_{2}}(M \times \mathbf{R})\right) .
$$

Furthermore, the asymptotic expansion of the local symbol $q$ of $Q$ can be calculated in terms of the asymptotic expansions of the local symbols $q_{i}$ of $Q_{i}$ by a formula reminiscent of the formula for the symbol of a composition for classical pseudodifferential operators. The principal symbol $q_{m_{1}+m_{2}}$ of $Q$ is $q_{1, m_{1}} \# q_{2, m_{2}}$. The recipe for \# is complicated and very technical. To calculate $\left(q_{1, m_{1}} \# q_{2, m_{2}}\right)(x, \sigma(x, \xi), \tau)$, one considers the corresponding invariant operators $\tilde{Q}_{1, m_{1}}$ and $\tilde{Q}_{2, m_{2}}$ on $H_{n} \times \mathbf{R}$, where $H_{n}$ is an osculating Heisenberg group. This is the appropriate analogue of "freezing coefficients". Then $q_{m_{1}} \# q_{m_{2}}$ is the principal symbol of the composition of the invariant operators, evaluated at the origin of $H_{n} \times \mathbf{R}$.

Because there is a symbol calculus, to construct the parametrix $Q$ for $\partial / \partial t+\square_{b}$, it suffices to find its principal symbol $q_{-2} \in S_{-2, h}$ locally. From this and the symbol calculus, we can obtain the full asymptotic expansion of the symbol $q$ of $Q$. Let $p_{2}$ denote the principal symbol of $\partial / \partial t+\square_{b}$. Then we are looking for a symbol $q_{-2}$ such that

$$
q_{-2} \# p_{2}=p_{2} \# q_{-2}=I \text {. }
$$

If we fix $x \in M$ and calculate in admissible coordinates centered at $x$, the Heisenberg operator corresponding to $p_{2}$ at $x$ is $\left(\partial / \partial t+\mathscr{L}_{n-2}\right) \cdot I$, so the Heisenberg operator corresponding to $q_{-2}$ at $x$ will be given by convolution with respect to Lebesgue measure on $H_{n} \times \mathbf{R}$ with the matrix-valued function $k$, which is 0 for $t<0$ and $2^{n} r_{t}^{n-2} \cdot I$ for $t>0$. Now $k$ vanishes for $t<0$ and is homogeneous of degree $-2 n-2$ with respect to the dilations $\lambda(u, z, t)=$ $\left(\lambda^{2} u, \lambda z, \lambda^{2} t\right)$. Hence its Fourier transform $\hat{k}$ is homogeneous of degree -2 with respect to the dilations (3.20) and extends to a holomorphic function on $\mathbf{R}^{2 n+1} \times \mathbf{C}_{-}$. In fact

$$
\hat{k}(0, \xi, \tau)=\int_{0}^{\infty} e^{-i \tau s-(n-2) \xi^{0} s} \cosh \left(\xi_{0} s\right)^{-n} \exp \left(-\left|\xi^{\prime}\right|^{2} \frac{\tanh \xi_{0} s}{\xi_{0}}\right) d s .
$$


Of course, admissible coordinates centered at $x$ will not be admissible coordinates centered at nearby points. To calculate $q_{-2}(x, \sigma(x, \xi), \tau)$ on a coordinate neighborhood $U$, we have to consider an appropriate transformation $\varphi$ from the given coordinates to admissible coordinates centered at $x$ and transform $\hat{k}$ under $\varphi^{-1}$. This calculation is tedious but not difficult, and does indeed result in a symbol $q_{-2}(x, \sigma(x, \xi), \tau) \in S_{-2, h}$.

Now we use our symbol calculus to obtain the full local asymptotic expansion of the symbol $q$ of $Q$. If we fix $x_{0} \in U$ and take as our coordinates on $U$ an admissible coordinate system centered at $x_{0}$, then the symbol calculus tells us that calculated in these coordinates, each term in the matrix of $q_{j}\left(x_{0}, \xi, \tau\right)$, for each $j \leqslant-2$, is a finite linear combination of terms of the form $g\left(x_{0}\right) h(\xi, t)$, where $h \in \mathscr{F}_{j, h}$ is independent of $x_{0}$, and $g$ may be computed by evaluating at $x_{0}$ a universal polynomial in derivatives of coefficients of the local orthonormal frame $\left\{X_{0}, Z_{j}, \bar{Z}_{j}\right\}$.

From the asymptotic expansion of the symbol of $Q$, we can read off information about the heat kernel $p(x, y, t)$. In particular, we obtain the analogue of the Minakshisundaram asymptotic expansion (1.9). In the case of a distinguished Levi metric, Webster [Web] constructed a canonical metric connection. This was generalized to arbitrary Levi metrics by C. M. Stanton [Stan]. We refer to this connection as the Webster-C. M. Stanton connection. As in the case of the Riemannian connection [ABP, Appendix II], if we use normal coordinates centered at $x_{0}$ and an orthonormal frame $\left\{Z_{j}\right\}$ which is parallel along geodesics through $x_{0}$, the coefficients in the Taylor series expansion of $X_{0}$ and the $Z_{j}$ about $x_{0}$ are polynomials in the curvature and torsion and their covariant derivatives evaluated at $x_{0}$. Because normal coordinates centered at $x_{0}$ are also admissible coordinates centered at $x_{0}$, this allows us to obtain a geometric interpretation of the asymptotic expansion, analogous to the McKean-Singer [MS] result in Riemannian geometry (see $\$ 1$ ). To state our result, we introduce the following notation. The map

is the composition of the maps

$$
\operatorname{tr}: \Lambda^{0,1} \otimes \Lambda^{1,0} \rightarrow \Lambda^{2 n+1}
$$

$$
I \otimes *: \Lambda^{0,1} \otimes \Lambda^{1,0} \rightarrow \Lambda^{0,1} \otimes \Lambda^{2 n} \text { and } \text { Alt: } \Lambda^{0,1} \otimes \Lambda^{2 n} \rightarrow \Lambda^{2 n+1},
$$

so

$$
\operatorname{tr} \mu \otimes \nu=\mu \wedge * \nu .
$$

THEOREM 3.4 [BGS]. On the diagonal of $M \times M$ the fundamental solution $p(x, y, t)$ of the heat equation for $\square_{b}$ has an asymptotic expansion

$$
\operatorname{tr} p(x, x, t) \sim t^{-n-1} \sum_{j=0}^{\infty} t^{j} K_{j}(x) d V(x)
$$

as $t \rightarrow 0$. The functions $K_{j}(x)$ are polynomials, depending only on $j$ and $n$, in the components of the curvature and torsion of the Webster-C. M. Stanton connection and their covariant derivatives evaluated in normal coordinates centered at $x$. In particular,

$$
K_{0}(x) \equiv \frac{n}{(2 \pi)^{n+1}} \int_{-\infty}^{\infty}\left(\frac{\tau}{\sinh \tau}\right)^{n} e^{-(n-2) \tau} d \tau
$$


For the special case of a distinguished Levi metric,

$$
K_{1}(x)=c(n) K(x),
$$

where $K$ is the scalar curvature of Webster's connection and $c(n)$ is a constant depending only on $n$.

As in many of the applications in Riemannian geometry, the last statement follows by using invariant theory to analyze $K_{1}$. For more general Levi metrics, $K_{1}$ also involves torsion and its derivatives. The result on $K_{0}$ also follows from (3.15), (3.17) and (3.18) and is equivalent to the Weyl-type theorem, Corollary 3.3.

M. Taylor has obtained a somewhat less precise asymptotic expansion using a different pseudodifferential operator calculus [T]. Tartakoff and I have obtained a new proof of the asymptotic expansion of Theorem 3.4 and of the general form of the coefficients $K_{j}$ from a very careful study of our formula (3.17) for the heat kernel [ST].

4. The heat equation for the $\bar{\partial}$-Neumann problem. In the previous section, I considered a problem on the boundary of a strictly pseudoconvex domain. Now I want to consider one on the domain itself. Let $\Omega$ be a bounded strictly pseudoconvex domain in $\mathbf{C}^{n+1}, n \geqslant 1$, with $C^{\infty}$ boundary $M$. Again, for simplicity I will restrict my attention to $(0,1)$ forms on $\Omega$. Let $u \in C^{\infty}\left(\Lambda^{0,1}(\bar{\Omega})\right)$. Then $u$ satisfies the $\bar{\partial}-$ Neumann boundary conditions if

$$
u_{\text {norm }}=0=(\bar{\partial} u)_{\text {norm }} \text { on } M .
$$

This is the analogue for $(0,1)$ forms of the classical Neumann boundary conditions. The $\bar{\partial}$-Neumann problem was introduced by Spencer in the early 1950s as a way of generalizing the Hodge Theorem and of solving the Levi problem. It is the following. Given a smooth $(0,1)$ form $f$ on $\bar{\Omega}$, prove existence and regularity of solutions $u$ of the equation

$$
\square u=f \text { on } \bar{\Omega}
$$

where $u$ satisfies the $\bar{\partial}$-Neumann boundary conditions (4.1). Here

$$
\square=\bar{\partial} \bar{\partial}^{*}+\bar{\partial} * \bar{\partial}=-\frac{1}{2} \Delta
$$

where $\Delta$ is the Euclidean Laplacian acting component by component. Unlike Dirichlet and Neumann boundary conditions, the $\bar{\partial}$-Neumann boundary conditions are not elliptic, so classical methods did not apply. Kohn solved the problem using $L^{2}$-methods [FK]. The fundamental solution $N$ of the $\bar{\partial}-\mathrm{Neu}$ mann problem is called the Neumann operator. The $(0,1)$ form $N f$ is the unique solution of

$$
\square N f=f, \quad N f \text { satisfies (4.1). }
$$

Again, I consider the corresponding heat equation,

$$
\begin{cases}(\partial / \partial t+\square) u=0 & \text { on } \Omega \times \mathbf{R}^{+}, \\ u(\cdot, t) \text { satisfies }(4.1) & \text { for each } t\end{cases}
$$


The initial value problem is to solve the heat equation with specialized initial value $u_{0} \in C\left(\Lambda^{0,1}(\bar{\Omega})\right)$, so the solution $u$ of (4.5) also satisfies

$$
\lim _{t \rightarrow 0} u(\cdot, t)=u_{0} .
$$

Unfortunately, because of the nonellipticity of the boundary condition, the classical methods for solving parabolic initial-boundary value problems-those used in [MS, Gr, Se 1 and Se 2] to construct the heat kernel with elliptic boundary conditions-do not apply. However, the heat operator, the fundamental solution of (4.5) and (4.6), is still given by integration against a smooth kernel [St 2]. Kohn proved the existence of a complete orthonormal set $\left\{\varphi_{i}\right\}$ of eigenforms for the $\bar{\partial}$-Neumann problem; the corresponding eigenvalues $\lambda_{i}$ are discrete and have finite multiplicity [FK]. The heat kernel is

$$
p(z, w, t)=\sum e^{-\lambda_{i} t} \varphi_{i}(z) \otimes \overline{\varphi_{i}(w)} .
$$

By Kohn's a priori estimates [FK], this kernel is smooth.

The problem of finding an explicit construction of $p$ is open. Even a first approximation is unknown. From an explicit construction of $p$, I hope one can obtain results similar to the ones I described in Riemannian and Hermitian geometry and for the $\bar{\partial}_{b}$-Laplacian. In particular, I hope there is an asymptotic expansion of

$$
\operatorname{tr} e^{-t \square}=\sum e^{-t \lambda_{i}}
$$

and a geometric interpretation of the coefficients. If there is, this and Karamata's Tauberian Theorem would give a new proof of Métivier's analogue of Weyl's Theorem.

THEOREM 4.1 (METIVIER [Me]). Let $N(\lambda)$ denote the number of eigenvalues of the $\bar{\partial}-$ Neumann problem on $(0,1)$ forms on $\Omega$ which are less than $\lambda$. Then

$$
N(\lambda) \sim\left(\frac{\mathrm{Vol} \Omega}{(2 \pi)^{n+1} \Gamma(n+1)}+\int_{M} c(z) d S\right) \lambda^{n+1}
$$

as $\lambda \rightarrow \infty$. Here, $d S$ is the induced volume element,

$$
c(z)=\frac{1}{2(2 \pi)^{n+1} \Gamma(n+2)} \sum_{j=1}^{n} \int_{0}^{\infty} e^{-t \mu_{j}} \prod_{j=1}^{n}\left(\frac{\tau \mu_{j}}{1-e^{-\tau \mu_{j}}}\right) d \tau,
$$

and $\mu_{j}$ are the eigenvalues of the Levi form at $z$ of a defining function $r$ with $|d r|=1$ on $M$.

The first term on the right side of (4.9) is the same as the first term for an elliptic boundary value problem for $\square$. The novel feature is that, because of the nonellipticity, the boundary influences the leading asymptotic behavior of the eigenvalues.

Another application of an explicit construction of $p$ would be a more explicit description of the Neumann operator (see (4.4)), since

$$
N=\int_{0}^{\infty} e^{-t \square} d t
$$


This is the analogue of (1.5) for the Green's operator. (In (1.5) I had to include a correction of $-H$. Here no correction is needed because the kernel of $\square$ on $(0,1)$ forms is trivial.)

Everything I have said until now in this section is for bounded domains. For general unbounded strictly pseudoconvex domains there is no theory of the $\bar{\partial}$-Neumann problem. In some cases one can impose appropriate conditions at infinity, together with the $\bar{\partial}$-Neumann boundary conditions, and obtain a theory. Even so, Kohn's work will not apply.

The one case where I have been able to construct the heat kernel explicitly is the Siegel domain $D$ of (3.1) [St 3]. This, of course, is unbounded; it is the strictly pseudoconvex analogue of the half space. The group law (3.11) of the Heisenberg group extends to a left action of the group on $D$. To exploit this, instead of the Euclidean metric I use an invariant metric, the one for which

$$
\begin{aligned}
& Z_{j}=\frac{\partial}{\partial z^{j}}+2 i \bar{z}^{j} \frac{\partial}{\partial w}, \quad j=1, \ldots, n, \\
& Z_{n+1}=i \sqrt{2} \frac{\partial}{\partial w},
\end{aligned}
$$

is an orthonormal basis of $T^{1,0}(\bar{D})$. This is a smooth metric on $\bar{D}$. Now the $\bar{\partial}$-Neumann problem is the following. Given $f \in C_{0}^{\infty}\left(\Lambda^{0,1}(\bar{D})\right)$, find a smooth solution $u$, vanishing at infinity, of (4.1) and (4.2), where norm and $\square$ are with respect to this metric. Again, I denote the solution by $N f$. Similarly, for the heat equation I require that my initial data $u_{0} \in C_{0}\left(\Lambda^{0,1}(\bar{D})\right)$, and the solution $u(\cdot, t) \in L^{2}\left(\Lambda^{0,1}(\bar{D})\right)$. Now the $\bar{\partial}$-Neumann problem becomes "simple". Let $\left\{\omega^{j}\right\}$ be the basis of $\Lambda^{1,0}(\bar{D})$ dual to $\left\{Z_{j}\right\}$. Then any $(0,1)$ form $f$ can be written as $f=\sum f_{j} \bar{\omega}^{j}$. The Laplacian $\square$ operates diagonally on the components of $f$. In addition, the $\bar{\partial}-$ Neumann boundary conditions are diagonal; they are

$$
\left.f_{n+1}\right|_{\partial D}=0,\left.\quad \bar{Z}_{n+1} f_{j}\right|_{\partial D}=0 \text { if } j \leqslant n .
$$

Thus, the initial value problem for the heat equation with $\bar{\partial}-\mathrm{Neumann}$ boundary conditions reduces to two problems on functions, one for the component $f_{n+1}$ and a second for the components $f_{j}, j \leqslant n$. The first has the classical Dirichlet boundary condition, and so is elliptic. The second, with the nonelliptic boundary condition, is more interesting. Both have explicit fundamental solutions or heat kernels. To give you an idea of what the solution looks like, I will just state the second problem and its solution. For an expository description of thie derivation of the kernels, see [St 5]; the details are in [St 3].

It is convenient to use as coordinates $z, u=\operatorname{Re} w$ and $r=-\rho$, where $\rho$ is the function of (3.3), so $D=\{r>0\}$. The second problem is the following. Given $f_{0}(z, u, r) \in C_{0}(\bar{D})$, find the square integrable solution $f$ of

$$
\begin{aligned}
& \left(\frac{\partial}{\partial t}+\mathscr{L}_{n-2}-\frac{1}{2}\left(\frac{\partial^{2}}{\partial u^{2}}+\frac{\partial^{2}}{\partial r^{2}}\right)\right) f=0, \quad r>0, \\
& \left(\frac{\partial}{\partial r}-i \frac{\partial}{\partial u}\right) f(z, u, 0, t)=0, \\
& \lim _{t \rightarrow 0} f(z, u, r, t)=f_{0}(z, u, r) .
\end{aligned}
$$


Here $\mathscr{L}_{n-2}$ is given by (3.6). The solution is

$$
f(z, u, r, t)=\int p\left(\left(z_{1}, u_{1}\right)^{-1}(z, u), r, s, t\right) f_{0}\left(z_{1}, u_{1}, s\right) d V
$$

where $\left(z_{1}, u_{1}\right)^{-1}(z, u)$ is Heisenberg multiplication and

$$
\begin{aligned}
& p(z, u, r, s, t)= \frac{1}{(2 \pi t)^{n+3 / 2}}\left(e^{-(r-s)^{2} / 2 t}+e^{-(r+s)^{2} / 2 t}\right) \\
& \times \int_{-\infty}^{\infty}\left(\frac{\tau}{\sinh \tau}\right)^{n} \exp \left(-i \tau \frac{u}{t}-\frac{|z|^{2} \tau}{t \tanh \tau}-\frac{\tau^{2}}{2 t}-(n-2) \tau\right) d \tau \\
&-\frac{1}{2^{n} \pi^{n+3 / 2} t^{n+2}} \int_{-\infty}^{\infty} \frac{\tau^{n+1}}{(\sinh \tau)^{n}} \quad \times \exp \left(\frac{-i \tau u}{t}-\frac{|z|^{2} \tau}{t \tanh \tau}-(n-2) \tau+\frac{(r+s) \tau}{t}\right) \\
& \quad \times\left(\int_{(r+s+\tau) / \sqrt{2 t}}^{\infty} e^{-\mu^{2}} d \mu\right) d \tau .
\end{aligned}
$$

Unfortunately, unlike the case of the $\bar{\partial}_{b}$-Laplacian, it does not seem possible to model the solution of the heat equation for the $\bar{\partial}$-Neumann problem (4.5) and (4.6) in general on the solution in the Siegel domain. However, I hope that the explicit formula of (4.16) will give some hint as to what would be a good first approximation to the general heat kernel.

As an application, I have shown that the Neumann operator $N$ for the $\bar{\partial}$-Neumann problem in the Siegel domain is the integral of the heat kernel with respect to $t$ [St 4]. This is the first explicit formula for the Neumann operator. Harvey and Polking [HP] and Range [Ran] have recently found explicit formulas for the Neumann operator of (4.4) in the unit ball in $\mathbf{C}^{n}$ and Kimura [Ki] has found a formula in the unit ball in $\mathbf{C}^{2}$.

\section{BIBLIOGRAPHY}

[ABP] M. Atiyah, R. Bott and V. K. Patodi, On the heat equation and the index theorem, Invent. Math. 19 (1973), 279-330; errata, ibid. 28 (1975), 277-280.

[BG] R. Beals and P. C. Greiner, Pseudodifferential operators associated to hyperplane bundles, Rend. Sem. Mat. Torino 41 (1983), 3-40.

[BGS] R. Beals, P. C. Greiner and N. K. Stanton, The heat equation on a CR manifold (preprint).

[B] M. Berger, Geometry of the spectrum. I, Proc. Sympos. Pure Math., vol. 27, Part 2, Amer. Math. Soc., Providence, R.I., 1975, pp. 129-152.

[Ca] T. Carleman, Propriétés asymptotiques des fonctions fondamentales des membranes vibrantes, Åttonde Skand. Matematikerkongressen (Stockholm, 1934), Ohlsson, Lund, 1935, pp. 34-44.

[Ch 1] J. Cheeger, Analytic torsion and the heat equation, Ann. of Math. (2) 109 (1979), 259-322.

[Ch 2] , On the spectral geometry of spaces with cone-like singularities, Proc. Nat. Acad. Sci. U.S.A. 76 (1979), 2103-2106.

[CL] S. Y. Cheng and P. Li, Heat kernel estimates and lower bound of eigenvalues, Comment. Math. Helv. 56 (1981), 327-338. 
[Dod] J. Dodziuk, Eigenvalues of the Laplacian and the heat equation, Amer. Math. Monthly 88 (1981), 686-695.

[Don] H. Donnelly, Minakshisundaram's coefficients on Kaehler manifolds, Proc. Sympos. Pure Math., vol. 27, Part 2, Amer. Math. Soc., Providence, RI, 1975, pp. 195-203.

[F] J. Fay, Analytic torsion and Prym differentials, Riemann Surfaces and Related Topics (Proc. 1978 Stony Brook Conf.), Ann. of Math. Stud., no. 97, Princeton Univ. Press, Princeton, N. J., 1981, pp. 107-122.

[FK] G. B. Folland and J. J. Kohn, The Neumann problem for the Cauchy Riemann complex, Ann. of Math. Stud., no. 75, Princeton Univ. Press, Princeton, N. J., 1972.

[FS] G. B. Folland and E. M. Stein, Estimates for the $\bar{\partial}_{b}$ complex and analysis on the Heisenberg group, Comm. Pure Appl. Math. 27 (1974), 429-522.

[Ga] M. P. Gaffney, Asymptotic distributions associated with the Laplacian for forms, Comm. Pure Appl. Math. 11 (1958), 535-545.

[Gi 1] P. Gilkey, Curvature and the eigenvalues of the Laplacian for elliptic complexes, Adv. in Math. 10 (1973), 344-382.

[Gi 2]_, Spectral geometry and the Kaehler condition for complex manifolds, Invent. Math. 26 (1974), 231-258.

[Gi Sa] P. Gilkey and J. Sacks, Spectral geometry and manifolds of constant holomorphic sectional curvature, Proc. Sympos. Pure Math., vol. 27, Part 2, Amer. Math. Soc., Providence, R. I., 1975, pp. 285-289.

[GW] C. S. Gordon and E. N. Wilson, Isospectral deformations of compact solvmanifolds, J. Differential Geom. 19 (1984).

[Gr St] P. C. Greiner and E. M. Stein, Estimates for the $\bar{\partial}-$ Neumann problem, Math. Notes, no. 19, Princeton Univ. Press, Princeton, N. J., 1977.

[Gr] P. Greiner, An asymptotic expansion for the heat equation, Arch. Rational Mech. Anal. 41 (1971), 163-218.

[HP] F. R. Harvey and J. C. Polking, The $\bar{\partial}-$ Neumann kernel in the ball in $\mathbf{C}^{n}$, Proc. Sympos. Pure Math., vol. 41, Amer. Math. Soc., Providence, R. I., 1984, pp. 117-136.

[I] A. Ikeda, On lens spaces which are isospectral but not isometric, Ann. Sci. École Norm. Sup. 13 (1980), 303-315. II, 1-23.

[Ka] M. Kac, Can one hear the shape of a drum?, Amer. Math. Monthly 73 (1966), no. 4, Part

[Ki] K. Kimura, Kernels for the $\bar{\partial}-$ Neumann problem on the unit ball, Thesis, Univ. of Toronto, 1983.

[Kr] S. Krantz, Function theory of several complex variables, Wiley, New York, 1982.

[LY] P. Li and S. T. Yau, On the Schrödinger equation and the eigenvalue problem, Comm. Math. Phys. 88 (1983), 309-318.

[Ma] S. Mandelstam, Dual resonance models, Phys. Rep. 13 (1974), 259-353.

[MS] H. P. McKean, Jr. and I. M. Singer, Curvature and the eigenvalues of the Laplacian, J. Differential Geom. 1 (1967), 43-69. 779-806.

[Me] G. Métivier, Spectral asymptotics of the $\bar{\partial}-$ Neumann problem, Duke Math. J. 48 (1981),

[MR] A. N. Milgram and P. C. Rosenbloom, Harmonic forms and heat conduction, Proc. Nat. Acad. Sci. U.S.A. 37 (1951), 180-184.

[Mil] J. Milnor, Eigenvalues of the Laplace operator of certain manifolds, Proc. Nat. Acad. Sci. U.S.A. 51 (1964), 542.

[Min] S. Minakshisundaram, Eigenfunctions on Riemannian manifolds, J. Indian Math. Soc. 17 (1953), 158-165.

[MP] S. Minakshisundaram and A. Pleijel, Some properties of the eigenfunctions of the Laplace operator on Riemannian manifolds, Canad. J. Math. 1 (1949), 242-256.

[Mu] W. Müller, Analytic torsion and R-torsion of Riemannian manifolds, Adv. in Math. 28 (1978), 233-305.

[Pa 1]V. K. Patodi, Curvature and the fundamental solution of the heat operator, J. Indian Math. Soc. 34 (1970), 269-285.

[Pa 2] _ Curvature and the eigenforms of the Laplace operator, J. Differential Geom. 5 (1971), 233-249.

[Pa 3] _ An analytic proof of the Riemann-Roch-Hirzebruch theorem for Kaehler manifolds, J. Differential Geom. 5 (1971), 251-273. 
[PI 1] A. Pleijel, $A$ study of certain Green's functions with applications in the theory of vibrating membranes, Ark. Mat. 2 (1952), 553-569.

[PI 2] __, On Green's functions and the eigenvalue distribution of the three dimensional membrance equation, Skand. Matematikerkongressen Lund, vol. 12, 1953, pp. 222-240.

[Q] D. Quillen, Informal lecture, Arbeitstagung, Bonn, 1983.

[Ran] M. Range, The $\bar{\partial}-$ Neumann operator on the unit ball in $\mathbf{C}^{n}$, Math. Ann. (to appear).

[Ray] D. B. Ray, Reidemeister torsion and the Laplacian on lens spaces, Adv. in Math. 4 (1970), 109-126.

[RS 1] D. B. Ray and I. M. Singer, R-torsion and the Laplacian on Riemannian manifolds, Adv. in Math. 7 (1971), 145-210.

[RS 2] __ Analytic torsion for complex manifolds, Ann. of Math. (2) 98 (1973), 154-177.

[Sch] A. S. Schwartz, The partition function of degenerate quadratic functional and Ray-Singer invariants, Lett. Math. Phys. 2 (1977/78), 247-252.

[Se 1] R. Seeley, Complex powers of an elliptic operator, Singular Integrals, Proc. Sympos. Pure Math., vol. 10, Amer. Math. Soc., Providence, R. I., 1967, pp. 288-307.

[Se 2] Analytic extension of the trace associated with elliptic boundary value problems, Amer. J. Math. 91 (1969), 963-983.

[Si] I. M. Singer, Eigenvalues of the Laplacian and invariants of manifolds, Proc. Internat. Congress Math. I (Vancouver, 1974), pp. 187-200.

[Stan] C. M. Stanton, Intrinsic connections for Levi metrics (in preparation). 547-578.

[St 1]N. K. Stanton, Holomorphic R-torsion for fiber bundles, Amer. J. Math. 100 (1978),

[St 2] , The fundamental solution of the heat equation associated with the $\bar{\partial}-N e u m a n n$ problem, J. Analyse Math. 34 (1978), 265-274.

[St 3] _ The heat equation for the $\bar{\partial}-$ Neumann problem in a strictly pseudoconvex Siegel domain. I, II, J. Analyse Math. 38 (1980), 67-112; 39 (1981), 189-202.

[St 4] _ The solution of the $\bar{\partial}-$ Neumann problem in a strictly pseudoconvex Siegel domain, Invent. Math. 65 (1981), 137-174.

[St 5] _ L'équation de la chaleur en plusieurs variables complexes, Séminaire Goulaouic-Meyer-Schwartz 1982-83, exposé VI.

[ST] N. K. Stanton and D. S. Tartakoff, The heat equation for the $\bar{\partial}_{b}$-Laplacian, Comm. Partial Differential Equations (to appear).

[T] M. Taylor, Noncommutative microlocal analysis. I, Mem. Amer. Math. Soc. (to appear).

[U] H. Urakawa, Bounded domains which are isospectral but not congruent, Ann. Sci. Ecole Norm. Sup. 15 (1982), 441-456.

[V] M. F. Vigneras, Variétés riemanniennes isospectrales et non isométriques, Ann. of Math. (2) 112 (1980), 21-32.

[Web] S. M. Webster, Pseudo-hermitian structures on a real hypersurface, J. Differential Geom. 13 (1978), 25-41.

[Wey] H. Weyl, Das asymptotische Verteilungsgesetz der Eigenwerte linearer partieller Differentialgleichungen (mit einer Anwendung auf die Theorie der Hohlraumstrahlung), Math. Ann. 71 (1912), 441-479.

DePartment of Mathematics, University of Notre Dame, Notre Dame, Indiana 46556 